\title{
CARCINOMA TÍMICO DE PADRÃO PAPILÍFERO: RELATO DE CASO EM PACIENTE HIV SOROPOSITIVO
}

\section{TYPICAL PAPILLARY STANDARD CARCINOMA: CASE REPORT IN HIV SOROPOSITIVE PATIENT}

Willian Moreira Machado'*, Amanda Poli Amhof', Guilherme Ribas Taques ${ }^{1,2}$, Fernando Luis Visoni Poliseli ${ }^{1,3}$

'Centro Universitário Campo Real. ${ }^{2}$ Universidade Estadual do Centro-Oeste do Paraná. ${ }^{3}$ Hospital de Caridade São Vicente de Paula.

*Autor correspondente: E-mail: willian_mmachado@hotmail.com ou med-willianmachado@camporeal.edu.br, tel: (42) 98403-8803

\section{RESUMO}

Timomas e carcinomas tímicos são tumores raros de células superficiais do órgão, sendo que as células tumorais no carcinoma do timo se desenvolvem mais rapidamente e podem se espalhar para outros locais do corpo com maior facilidade comparadas com demais neoplasias mediastinais, em que timomas, carcinomas e carcinoides tímicos são as mais comuns e mais agressivas. Sabe-se, hoje, que pessoas infectadas pelo HIV possuem maior suscetibilidade em desenvolver câncer. Este relato de caso mostra as características da apresentação de um carcinoma tímico em paciente soropositivo, relatando a rara incidência da neoplasia de timo de padrão papilífero, destacando a importância e as dificuldades quando se avaliam portadores de doenças raras.

Palavras-chave: carcinoma de padrão papilífero, câncer tímico e carcinoma tímico metastático.

\section{ABSTRACT}

Thymomas and thymic carcinomas are rare tumors of superficial cells of the organ, and the tumor cells in thymus carcinoma develop more quickly and can spread to other parts of the body more easily compared to other mediastinal neoplasms, in which thymomas, carcinomas and thymic carcinoids are the most common and most aggressive. It is now known that people infected with HIV are more susceptible to developing cancer. This case report shows the characteristics of the presentation of a thymic carcinoma in a seropositive patient, reporting the rare incidence of papillary thymus neoplasia, highlighting the importance of the difficulties when evaluating patients with rare diseases.

Keywords: papillary pattern carcinoma, thymic cancer and metastatic thymic carcinoma.

\section{INTRODUÇÃO}

O timo é um órgão linfocitário bilobado que desempenha papel importante no sistema imune. O timo consiste em dois lobos laterais mantidos em estreito contato por um tecido conjuntivo. 
Histologicamente, este tecido conjuntivo divide o parênquima do órgão em lóbulos incompletos. Cada lóbulo possui uma parte periférica denominada zona cortical, que envolve a parte central, a zona medular. Nesta zona medular se encontram os Corpúsculos de Hassall, uma característica similar as regiões é que essas possuem o mesmo tipo celular, os quais são os linfócitos T e células epiteliais.

Sabe-se que, com o passar dos anos, o timo sofre uma atrofia e é, gradualmente, substituído por tecido adiposo. O timo está localizado na porção superior do mediastino anterior durante a infância, enquanto no adulto, sua localização é incerta em função da atrofia. Situa-se, parcialmente, no tórax e no pescoço se estendendo desde a quarta cartilagem costal até o bordo inferior da glândula tireoide. Normalmente, o lobo direito é maior que o esquerdo.

Sua principal função é produção e maturação de linfócitos T durante a infância. Em seguida, os linfócitos migram para o sistema linfático auxiliando o sistema imunológico, principalmente, contra vírus e fungos.

Os tumores de timo são raros e, especificamente, o carcinoma tímico compreende menos de 10\% dos tumores dessa glândula, acometendo, principalmente, pessoas entre 40 e 60 anos. As neoplasias epiteliais de timo incluem timomas, carcinoides e carcinoma tímico. Em função da escassez de dados na literatura sobre os vários aspectos desta patologia, principalmente, a sua associação com pacientes HIV soropositivos fica evidente a necessidade de mais estudos e discussões sobre o tema. O objetivo deste trabalho é relatar um caso de carcinoma tímico em paciente soropositivo, em decorrência da rara incidência da neoplasia de timo de padrão papilífero.

\section{CASO}

Paciente do sexo feminino, afrodescendente, com 50 anos, encaminhada da Unidade Básica de Saúde (UBS) ao Hospital de referência do município de Guarapuava - PR, em outubro de 2018, para avaliar queixas relatadas. Na UBS, a paciente relatou dispneia e cansaço persistente a pequenos esforços, além de desconforto retroesternal recente, sem história de tosse, expectoração. Nega anormalidades em antecedentes familiares e em seus antecedentes pessoais, relata ser HIV soropositivo, episódio de colelitíase e uma constatação histológica para processo linfoproliferativo atípico em região mediastinal, sem evidenciar evolução (detectado por avaliação histológica em 2008). Relata alergia à diclofenaco e nega tabagismo e etilismo. Ao exame físico, apresentava regular estado geral, sem demais alterações sistemáticas.

Por solicitação do clínico geral, a paciente realizou Raio-X de tórax e eletrocardiograma. O ECG não apresentou anormalidades, enquanto no Raio-X foi observada uma massa em mediastino anterior e líquido no pulmão esquerdo. Com esses achados clínicos, a paciente foi encaminhada para o centro de especialidades oncológicas do município de Guarapuava. No setor de oncologia do Hospital foi realizada biópsia e análise patológica da massa mediastinal, que sugeriu linfonodo cervical - carcinoma de padrão papilífero metastático para linfonodo.

Os achados clínicos da paciente estão relacionados com o quadro detectado em 2008. Neste ano, foi a primeira vez que a paciente soube da anormalidade em seu corpo. O Raio-X evidenciou a presença de uma massa em região mediastinal. Vale ressaltar a ausência de qualquer queixa de dor por parte da paciente. Uma tomografia e posterior biópsia (transtorácica com agulha) mostrou um processo linfoproliferativo atípico, uma lesão sólida de $6,5 \mathrm{~cm}$ em mediastino anterior, tendo sido 
sugerido um linfoma ou timoma via análise histopatológica. A conduta médica do caso se restringiu a acompanhamento sem necessidade de novas investigações.

Além disso, no ano de 2008, foi realizada uma endoscopia respiratória com coleta de lavado brônquico, que apresentou laudo negativo para malignidade. Também, foi realizado uma ultrassonografia de abdome após relatar dor em hipocôndrio direito. O exame indicou colelitíase (cálculo único e móvel de aproximadamente $13 \mathrm{~mm}$ ) e imagem cística septada do fígado.

Exames laboratoriais solicitados, em 2009, detectaram que a paciente é HIV soropositivo. Esses achados corroboram com a literatura, pois se sabe que pacientes soropositivos possuem uma maior suscetibilidade em desenvolver câncer. Em anamnese, relatou não ter apresentado sintomatologias que pudessem indicar uma imunodeficiência. A paciente afirma ter contraído a doença via relação sexual. Com a carga viral alta e CD4 baixo, iniciou o tratamento com antirretrovirais (Lamivudina, Efavirenz). De acordo com a doente, a transmissão do HIV ocorreu anos antes do primeiro diagnóstico da presença da massa mediastinal. Até o momento nem a paciente, nem os médicos sabiam da doença.

No ano de 2012, foi detectada a necessidade da colecistectomia. A cirurgia transcorreu sem anormalidades, bem como o pós-operatório. Pela época não foi correlacionada nenhuma associação entre a massa mediastinal e o fator HIV soropositivo.

A partir de outubro de 2018, com a história pregressa observada e os dados recém coletados houve o início de um diagnóstico mais preciso para uma possível neoplasia. No laudo da tomografia de tórax se constatou um tumor em mediastino anterior com metástase pleural e derrame pleural volumoso (carcinoma tímico pouco diferenciado infiltrando tecido fibroconjuntivo).

Em novembro, uma videotoracostomia e pleurodese a esquerda com drenos foram realizados. (Drenos oscilates, débito de líquido de amarelo citrino, sem escape de ar, aderência de pleura multisseptada). Em uma nova TAC de tórax e abdome se observou um espessamento pleural basal, bilateral, com pequeno derrame pleural a esquerda, linfonodo aumentado com 15mm de diâmetro, em situação para-aórtica esquerda, na altura da emergência da artéria mesentérica superior. Além disso, diagnosticou-se lesão expansiva em mediastino anterior de 11,5cm, lesão axilar esquerda e linfonodomegalia cervical esquerda. Pequeno pneumotórax à esquerda, com dreno torácico desse lado.

Em acompanhamento, novos exames em dezembro registraram aumento da área cardíaca, ectasia difusa da aorta, opacidade macronodular na região hilar do pulmão esquerdo, lesão pleuro-parenquimatosa na base pulmonar esquerda, hiperinsulflação do pulmão direito e os aspectos radiográficos sugestivos para neoplasia pulmonar, com atelectasia de parte do lobo inferior do pulmão esquerdo. A partir desses resultados, a paciente iniciou a quimioterapia sem interrupção dos medicamentos antirretrovirais.

Uma biopsia foi solicitada em 2019, constatando um carcinoma de padrão papilífero metastático para linfonodo. Foi a primeira vez do diagnóstico de câncer específico. Recomendou-se, a critério clínico, complementação diagnóstica com exame de imuno-histoquímica.

A partir de um exame físico, em março de 2019, uma ultrassonografia de tireoide mostrou um nódulo hipocogênico de 14mm, limites irregulares, hipervascularizado ao Doppler, linfonodomegalia adjacente ao lobo esquerdo, linfonodo jugular direito (Tireoglobulina = 26 ng $/ \mathrm{ml}$ ). 
Figura 1 - Raio-X AP evidenciando massa em mediastino anterior.

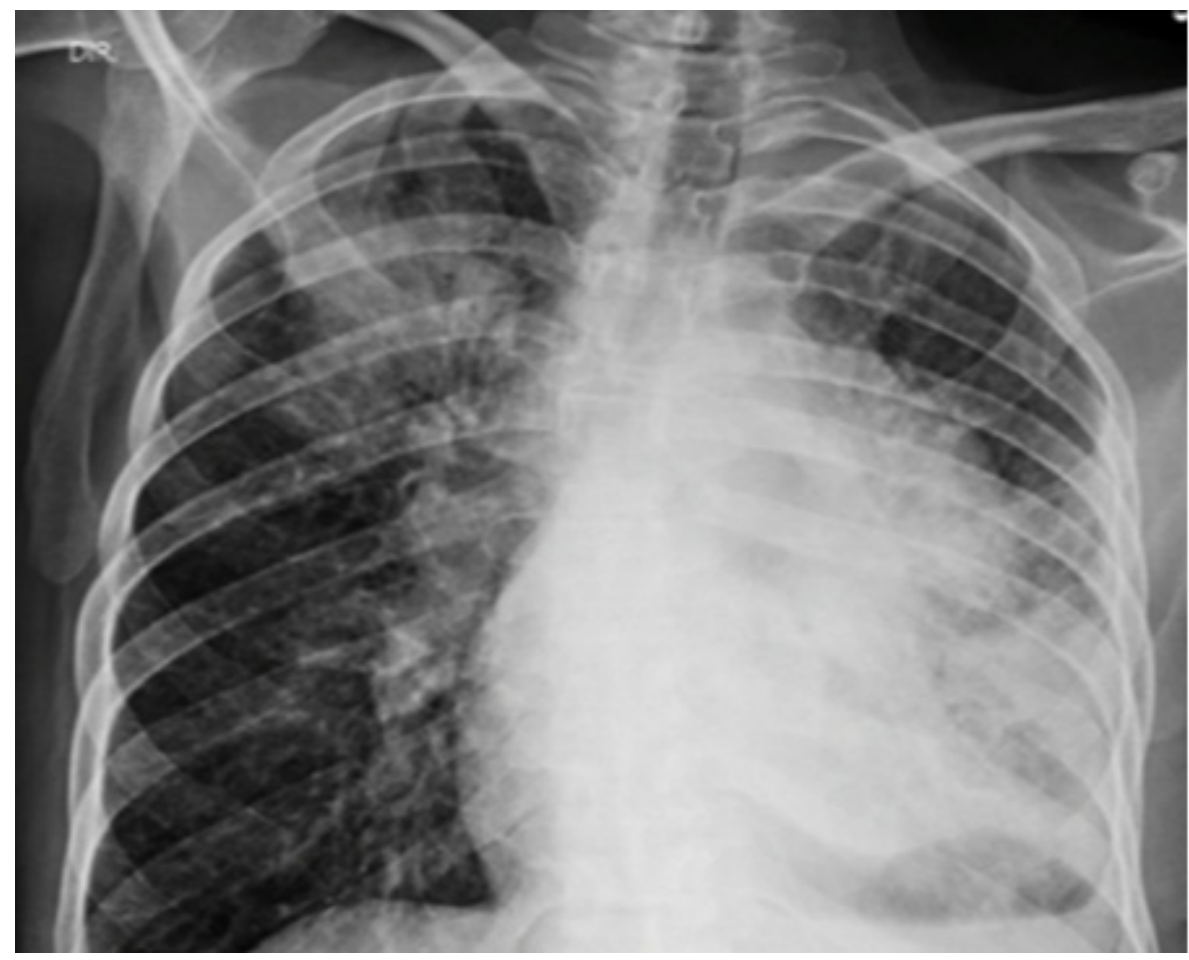

Uma nova tomografia de tórax, realizada para controle do carcinoma tímico, mostrou que a massa heterogênea havia passado para $12,6 \mathrm{~cm}$ de diâmetro, ou seja, um aumento de $1,1 \mathrm{~cm}$ em relação a TAC anterior $(11,5 \mathrm{~cm})$, realizada em novembro de 2018. A infiltração mediastinal envolveu aorta, vasos pulmonares, traqueia e brônquios principais. Moderado derrame pleural à esquerda, determinando atelectasias compressivas, também foi observado. Além disso, nota-se espessamento dos septos interlobulares no parênquima do hemitórax esquerdo e uma fratura compressiva de aspecto patológico no corpo vertebral T5, por possível acometimento secundário. Como diagnóstico, conclui-se tratar de um carcinoma metastático para pleura com células papilíferas (Câncer tímico com variante papilar). 
Figura 2 - Tomografia de tórax evidenciando calcificações no pulmão esquerdo, atelectasia de brônquios e massa heterogênea.

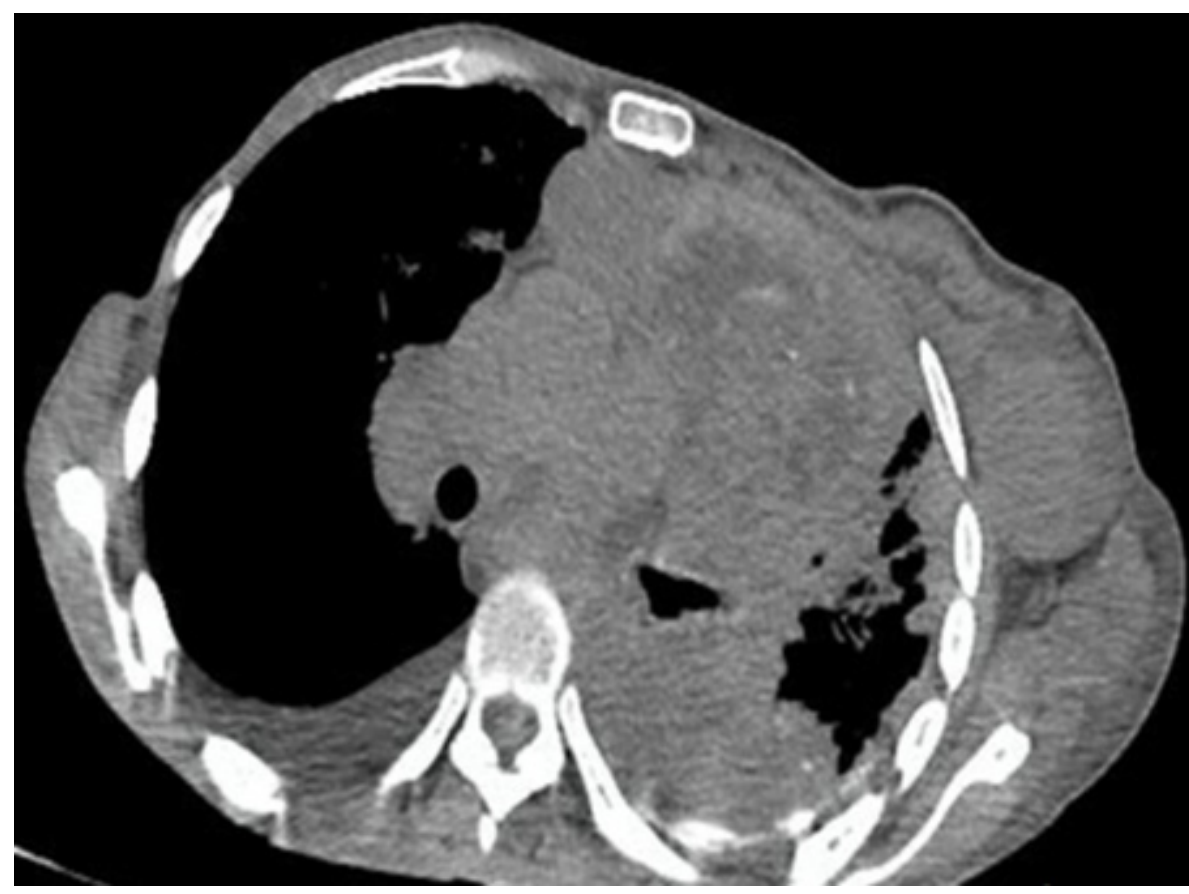

Na recolha analítica dos hemogramas, do primeiro semestre de 2019, foram observados alguns dados relevantes. A realização dos exames ocorreu nos meses de janeiro, março, maio e junho. Observou-se alteração, em suas hemácias, no mês de junho de 2,95 milhões/ $\mu \mathrm{L}$. Os valores de VCM e HCM se mostraram alterados em todos os exames, conforme sequência cronológica: VCM (106.2; $109.6 ; 113 ; 107.4 \mathrm{fL})$ e HCM $(36.7 ; 38.2 ; 38.5 ; 36.9$ pg). Leucopenia nos meses de janeiro, março e maio $(3700 ; 3500 ; 4640 \mu \mathrm{L})$. E, alterações em linfócitos típicos nos meses de janeiro, maio e junho (44\%; 21\%; 16\%). Nesse sentido, fica evidente uma piora no quadro hematológico da paciente.

Para fechamento do caso da paciente, a imuno-histoquímica afirmou ser compatível com carcinoma pouco diferenciado infiltrando tecido fibroconjuntivo e favorece carcinoma tímico. Os anticorpos positivos encontrados foram: CD5 (++/3), CK7 (+/3) e Pan CK AE1/AE3 (+++/3).

Figura 3 - Análise patológica H/E 400x evidenciando padrão papilífero em carcinoma metastático.

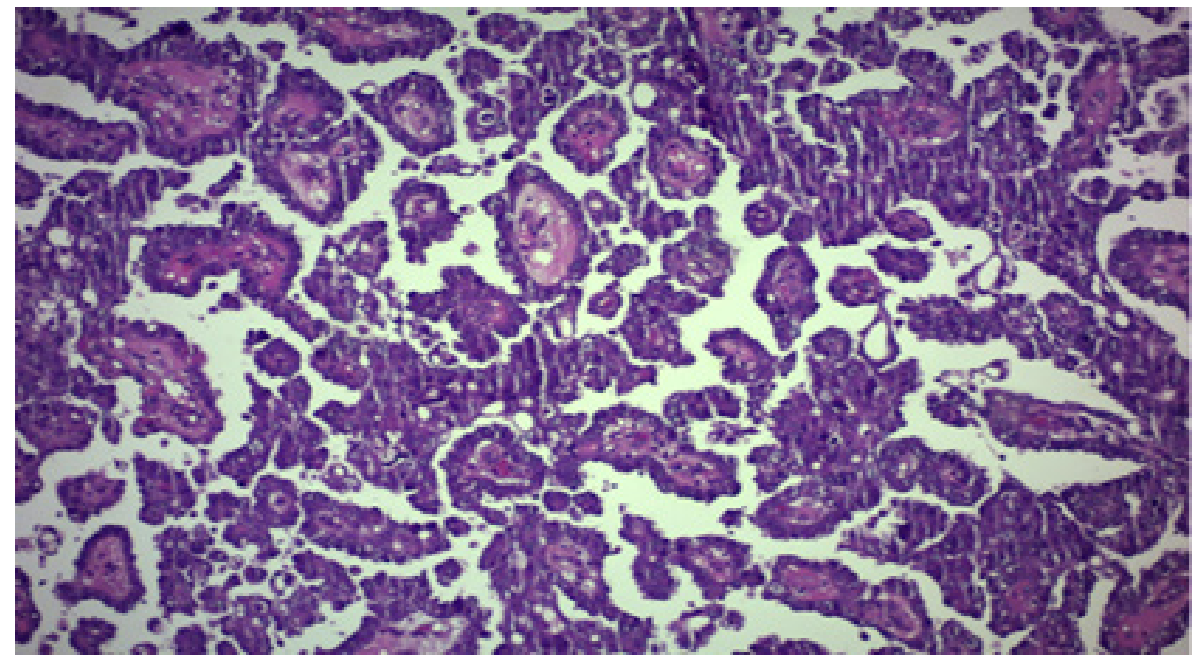


Figura 4 - Análise imuno-histoquímica com marcador CK AEl/AE3.

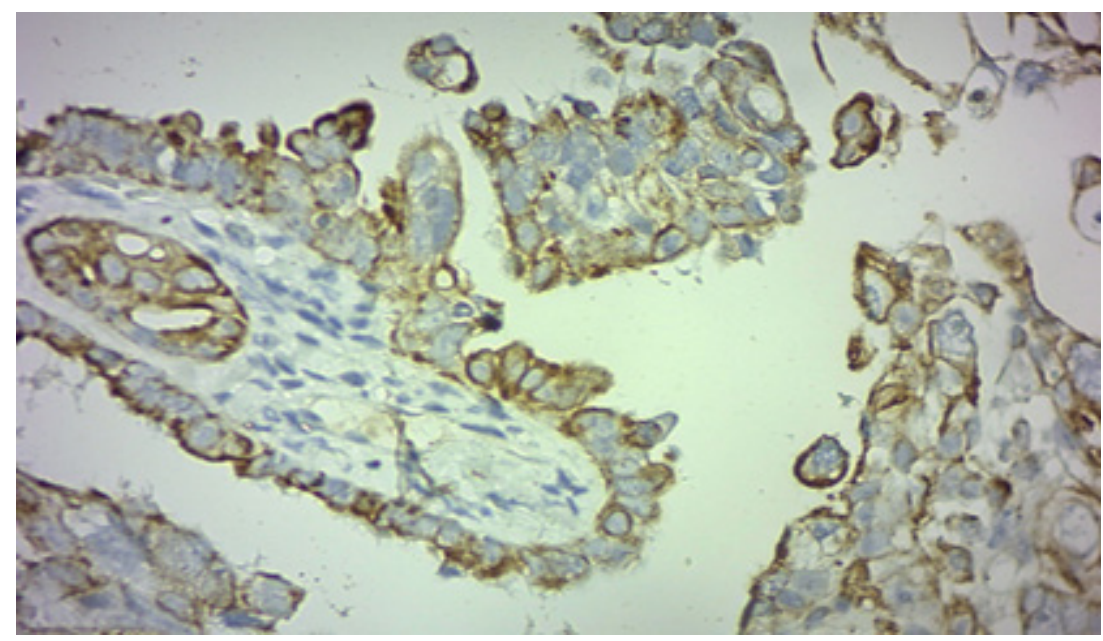

No mês de julho, uma tomografia de tórax realizada identificou uma extensão da lesão mediastinal, região hilar pulmonar com uma massa ganglionar axilar esquerda e, ainda, a presença de lesão osteolítica em T5. Como diagnóstico se concluiu que o carcinoma havia se espalhado definindo-o como carcinoma metastático para pleura. Ainda, neste mês, a paciente foi submetida a uma punção pulmonar para a retirada de $150 \mathrm{~mL}$ de secreção. A partir do mau prognóstico e da baixa resposta foi suspendida a quimioterapia. Para alívio das dores foram prescritos analgésicos não opioides e também opioides e para inibição da tosse antialérgicos associados com a codeína se necessário. A paciente segue em acompanhamento psicológico.

A paciente veio a óbito em setembro de 2019. Vale salientar que ela continuou sendo vigiada em consultas de oncologia médica, apresentando evoluções clínicas e físicas em função da metástase. O tratamento antirretroviral persistiu, sem manifestação de sintomatologias da doença. Foi realizado acompanhamento psicológico com frequência em decorrência do mau prognóstico concluído pela junta médica.

\section{DISCUSSÃO}

A evolução do carcinoma tímico, muitas vezes, é imprevisível, variando desde assintomática até a possibilidade de disseminação intra e extratorácica. Histologicamente, o timoma e o carcinoma tímico são os subtipos mais frequentes e surgem apenas nas células epiteliais do timo (VENUTA F et al, 2012). Os carcinoides tímicos têm taxa de sobrevida em 5 anos de $60 \%$ para tumores localizados, 50\% para regional e 30\% para metástase a distância. Dessa forma, o estadiamento baseado na extensão anatômica do tumor é um dos principais determinantes do prognóstico (AHN L et al, 2012). A apresentação clínica mais frequente diz respeito a manifestações relacionadas com efeito de massa local (tumores entre $6-20 \mathrm{~cm}$ de maior diâmetro), sendo os sintomas mais comuns a dor ou desconforto torácico, tosse, dispneia ou síndrome da veia cava superior (CARVALHO L et al, 2010 e DETTERBECK FC, ZEECHAN A, 2013).

Estes dados mostram dados semelhantes, nos quais a TC mostrou que uma massa heterogênea havia passado para 12,6 cm de diâmetro, ou seja, um aumento de 1,1 cm em relação a TAC anterior $(11,5 \mathrm{~cm})$. A infiltração mediastinal envolveu aorta, vasos pulmonares, traqueia e brônquios principais. Tratando-se de uma doença com grande progressão local possível, recidiva e metastização em elevada 
percentagem de casos, apresenta um mau prognóstico geral. Contagens altas de neutrófilos e PCR com história de tabagismo também podem mostrar um estágio avançado da doença. A associação entre marcadores inflamatórios e estágio avançado foi consistente com relatórios recentes (YANAGIYA M et al, 2018; MURIANA P et al, 2018).

Fica evidenciado que a imuno-histoquímica e a análise patológica foram de fundamental importância para o desfecho do quadro. A positividade dos marcadores CD5, CK7 e Pan CK AE1/AE3 nortearam que se tratava de um carcinoma tímico. E a análise patológica evidenciou o padrão papilífero em carcinoma metastático. O Pan Ck AE1/AE3, principalmente, por ser capaz de reconhecer polipeptídios de citoqueratina de quase todos os epitélios, sendo útil para diferenciar tumores epiteliais de tumores não epiteliais, sendo a sua marcação, geralmente, negativa para os últimos. Os carcinomas tímicos são caracterizados por sua variabilidade histológica e, frequentemente, semelhante a tumores vistos em outros sistemas de órgãos. Tentativas têm sido feitas para investigar as características moleculares desses tumores, na esperança de que o perfil molecular possa ser usado para prever o prognóstico ou levar ao desenvolvimento de novas estratégias de tratamento (WEISSFERD A, WISTUBA I, MORAN C, 2012).

A infecção pelo HIV predispõe ao câncer. Dados publicados mostram que a incidência de malignidade é de $4 \%$ ao ano. Foi levantada a hipótese de que a supressão de células T permita um crescimento desregulado de linhas celulares infectadas por Epstein Barr sobre outras linhas celulares (McDonald, M et al, 2005; BIGGAR RJ, FRISCH M, GOEDERTJJ, 2000). No estudo de Hazra e Mackall, em 2005, foi avaliado como possível mecanismo da falha na reconstituição imunológica no HIV a infecção associada à falha do timo. Neste trabalho se conclui que a interleucina (IL)-7, uma citocina com papéis críticos na timopoese, é elevada durante linfopenia, reportada neste estudo, e tem sido sugerido como um possível modulador da reserva tímica. O estágio da doença em si é importante, porque adultos com contagem de células CD4+ superiores a 200 antes do tratamento demonstram maiores quantidades de CD4+ (HAZRA R. e MACKALL C, 2005 e WHILSHIRE et al, 2016).

Em 2013, Leathers et al apresentaram um caso de um adulto com a coexistência incomum de timoma e HIV, complicados por infecções oportunistas. Sabe-se que o HIV e o timoma afetam a sistema imunológico, mas o efeito de sua coexistência na função imunológica ainda está sendo estudado. Infecções oportunistas na ausência de linfopenia podem sugerir a presença de um defeito na imunidade celular. O paciente apresentou algumas infecções oportunistas, o que corrobora com os dados deste autor (LEATHERS, $\mathrm{C}$ et al, 2013).

Para a maioria dos tumores tímicos, a cura ocorre através da cirurgia associada com a radioterapia. Cerca de um terço dos pacientes apresentam tumores em estágio já avançado, sendo necessário quimioterapia (BERGHMANS T, 2018). O carcinoma tímico é considerado uma doença rara, tendo uma agressividade maior comparado ao timoma, com isso, um péssimo prognóstico. A relativa raridade desses tumores torna a avaliação clínica difícil. Com isso, a análise molecular é a forma promissora para avaliação (KRISHNAN, M e GANTI, AK, 2019).

Dados do banco de dados de Estatísticas do Câncer dos Estados Unidos mostraram um aumento no diagnóstico de câncer tímico, que é atribuído, principalmente, ao aumento significativo na incidência de timoma e carcinoma tímico particularmente em mulheres. A incidência de carcinoma tímico aumentou de 2004 a 2015 (HSU, CH, et al, 2019). Alguns trabalhos sobre quimioterapia em pacientes com HIV sugerem que as respostas são ruins, o tempo de sobrevida é curto e a toxicidade é maior comparado com pacientes HIV negativos. Um dos primeiros trabalhos a publicar sobre este 
assunto mostrou que a quimioterapia com dois medicamentos não pareceu induzir a replicação viral do HIV, e foi sugerido que se possa diminuir a terapia retroviral concomitante para administrar mais quimioterapia mielossupressora (GONZALEZ C et al, 2000; THOMAS C, 1999). A cirurgia é a base do tratamento com uma sobrevida proporcional aos estágios. A quimioterapia à base de platina continua sendo o padrão de tratamento para pacientes com doença avançada (SCORSETTI, M et al, 2016). Com isso, este caso sugere que os carcinomas tímicos, no cenário do HIV, podem responder, inicialmente, aos quimioterápicos, já que o paciente não apresentou efeitos adversos do uso destas terapias.

\section{CONSIDERAÇÕES FINAIS}

Estes dados mostram que a evolução do quadro neoplásico da paciente se mostrou correlativo com a soropositividade para HIV. Dessa forma, fica evidente que os 10 anos entre a descoberta da massa mediastinal até a conclusão de um carcinoma tímico metastático de padrão papilífero ocorreram de forma evolutiva. Em função da raridade do caso e a baixa incidência de pacientes com neoplasias tímicas, novos estudos devem ser realizados para aprofundamento do assunto, uma vez que a literatura é escassa neste tema. O relato de carcinoma tímico em paciente soropositivo fica evidenciado, principalmente, pela imunohistoquímica e de forma complementar com os dados hematológicos, raio-x e tomografias.

\section{REFERÊNCIAS}

VENUTA F, et al. Thymoma and thymic carcinoma. Gen Thorac Cardiovasc Surg, 2012. Disponível em: https://pubmed.ncbi.nlm.nih.gov/22237733/.

AHN S, Lee JJ, HA SY, SUNG CO, KIM J, HAN J. Clinicopathological analysis of 21 thymic neuroendocrine tumors. Korean J Pathol, 2012. Disponível em: https://www.ncbi.nlm.nih.gov/pmc/ articles/PMC3479770/.

CARVALHO L, et al. Tumor carcinoide do timo - caso clínico. Revista Portuguesa de

Pneumologia, 2010. Disponível em: http://www.scielo.mec.pt/scielo.php?script=sci_ arttext\&pid=S0873-21592010000100013

DETTERBECK FC, ZEECHAN A. Thymoma: Current diagnosis and treatment. Chin Med J (Engl). 2013. Disponível em: https://insights.ovid.com/pubmed?.pmid=23769581

MURIANA P, et al. Assessment of the prognostic role of neutrophil-to-lymphocyte ratio following complete resection of thymoma. J Cardiothorac Surg, 2018. Disponivel em: https://www.ncbi.nlm. nih.gov/pmc/articles/PMC6245904/

YANAGIYA M, et al. Prognostic significance of the preoperative neutrophil- to-lymphocyte ratio for complete resection of thymoma. Surg Today, 2018. Disponível em: https://pubmed.ncbi.nlm.nih. gov/29063371/

WEISSFERD A, WISTUBA I, MORAN C. Molecular aspects of thymic carcinoma. Lung Cancer, 2012. Disponível em: https://pubmed.ncbi.nlm.nih.gov/22921473/.

MCDONALDS M, et al. Thymic Carcinoma in a Child With HIV Infection. Wiley-Liss, 2005. Disponível em: https://onlinelibrary.wiley.com/doi/abs/10.1002/pbc.20694 
BIGGAR RJ, FRISCH M, GOEDERT JJ. Risk of cancer in children with AIDS. JAMA, 2000. Disponível em: https://jamanetwork.com/journals/jama/fullarticle/vol/284/pg/205

HAZRA R, MACKALL C. Thymic Function in HIV Infection. Curr HIV/AIDS Rep, 2005. Disponível em: https://link.springer.com/article/10.1007/s1 1904-996-0005-2.

WILSHIRE CL, et al. Robotic Resection of $3 \mathrm{~cm}$ and Larger Thymomas Is Associated With Low Perioperative Morbidity and Mortality. Innovations (Phila). 2016. Disponível em: https://doi. org/10.1097/imi.0000000000000295

LEATHERS, CA, et al. Opportunistic infections in a patient with HIV and thymoma. J Allergy Clin Immunol, 2013. Disponível em: https://linkinghub.elsevier.com/retrieve/pii/S2213-2198(13)00194-3.

BERGHAMNS T, et al. Systemic treatments for thymoma and thymic carcinoma: A systematic review. Lung Cancer, 2018. Disponível em: https://pubmed.ncbi.nlm.nih.gov/30527189/.

KRISHNAN, M E GANTI, AK. The role of targeted therapy in thymic carcinoma. J Oncol Pharm Pract, 2019. Disponível em: https://pubmed.ncbi.nlm.nih.gov/31159657/.

$\mathrm{HSU} \mathrm{CH}$, et al. Trends in the incidence of thymoma, thymic carcinoma, and thymic neuroendocrine tumor in the United States. PLoS One, 2019. Disponivel em: https://pubmed.ncbi.nlm.nih. gov/31891634/.

GONZALEZ CE, et al. Impact of chemotherapy for AIDS-related malignancies in pediatric HIV disease. AnnNYAcad Sci, 2006. Disponível em: https://www.researchgate.net/ publication/228047841_Impact_of_Chemotherapy_for_AIDS-Related_Malignancies_in_Pediatric_ HIV_Disease

THOMAS CR, et al. Thymoma: State of the art. J Clin Oncol, 1999. Disponível em: https://ascopubs. org/doi/10.1200/JCO.1999.17.7.2280?url_ver=Z39.88-2003\&rfr_id=ori:rid:crossref.org\&rfr_dat=cr_ pub\%20\%200pubmed.

SCORSETTI M, et al. Thymoma and thymic carcinomas. Crit Rev Oncol Hematol, 2016. Disponível em: https://pubmed.ncbi.nlm.nih.gov/26818050/. 